\title{
A Method for Signal Coordination in Large-Scale Urban Road Networks
}

\author{
Bao-Lin Ye, Weimin Wu, and Weijie Mao \\ National Laboratory of Industrial Control Technology, Institute of Cyber-Systems and Control, Zhejiang University, \\ Yuquan Campus, Hangzhou, Zhejiang 310027, China \\ Correspondence should be addressed to Weimin Wu; wmwu@iipc.zju.edu.cn
}

Received 28 October 2014; Accepted 5 January 2015

Academic Editor: Kun Liu

Copyright (C) 2015 Bao-Lin Ye et al. This is an open access article distributed under the Creative Commons Attribution License, which permits unrestricted use, distribution, and reproduction in any medium, provided the original work is properly cited.

\begin{abstract}
Signal coordination methods have been widely investigated to improve the performance of traffic control system in urban road networks. Due to the large-scale of road networks and numerous time-varying parameters, a basic challenge faced by these methods is the high computational complexity. In this research, a method to optimize and coordinate the traffic signal in urban road networks is developed, which deals with this challenge by decomposing the network into several arterial roads and scattered intersections. As a result, an area coordination problem has been solved by coordinating several principal arterial roads and isolated intersections. Firstly, we propose a bandwidth oriented two-way arterial coordination approach. In contrast to previous methods, one important feature of the proposed approach is that not only the phases which provide right of way to the coordinated directions but the phases which provide right of way to the uncoordinated directions are considered. Secondly, to reduce the computational complexity a network decomposition approach is designed, in which a priority level index is defined to quantify the priority level of each road. Then, a coordination algorithm for the whole road network is developed. Finally, effectiveness and efficiency of the proposed method are validated by simulations on VISSIM.
\end{abstract}

\section{Introduction}

Aterial traffic congestion and the associated time-consuming, traffic jams, and noise and air pollution are a hard nut to crack to traffic administration organs of both the developed and developing countries. Consequently, the researches on reducing urban traffic congestion with advanced control and information technologies have long been a hot research topic. It is generally recognized that traffic signal control is one of the most effective and efficient manners to alleviate traffic congestion. As a result, many traffic signal control strategies have been developed [1-25].

As one of the earliest signal control strategies, isolated intersection control strategies mainly focused on the optimization of an isolated intersection. In general, it includes fixed-time control and traffic-responsive control [1]. The former one is based on the statistics of history traffic flow patterns. The latter one is an online process that utilizes real time traffic data to optimize the green time of a traffic light. As pointed out in [2], with a strong optimization capability, the isolated intersection control strategies can provide a strong basis for implicit coordination with the adjacent intersections. Hence, several new signal control strategies for isolated intersection have been proposed. For example, a new approach for controlling the traffic at isolated intersections is developed, whereby the dynamic behavior of the traffic is considered and modeled with Petrinets [3]. In [4], inspired by the observation of self-organized oscillations of pedestrian flows at bottlenecks, Lämmer and Helbing proposed a selforganization approach to traffic light control. Xie et al. [5] reported on a schedule-driven intersection control strategy, whereby the main characteristic is an alternative formulation of intersection control optimization as a scheduling problem to reduce the state space.

Then, to improve the performance of isolated intersection control, coordinated control strategies were proposed. In general, coordinated control mainly falls into two categories, fixed-time coordinated control and coordinated trafficresponsive strategies. For example, bandwidth oriented signal timing approaches MAXBAND [6] and MULTIBAND [7] 
and traffic network study tool TRANSYT [8] are well known fixed-time coordination control methods. SCOOT [9] and SCATS [10] are regarded as the earliest and leading examples of the coordinated traffic-responsive control approaches. After that, lots of model-based traffic-responsive control strategies with rolling time horizon emerged in 1990s, for example, OPCA [11], PRODYN [12], and CRONOS [13]. However, the performance of these strategies is limited due to the fact that the computational complexity grows exponentially with the size of the urban traffic network. Moreover, based on store-and-forward strategy, a trafficresponsive urban control (TUC) framework was proposed in [14]. Despite the simplicity and the efficiency of the proposed TUC, such a framework does not allow for immediate consideration of modifications and expansions of the controlled traffic network.

Owing to the fact that the predominant traffic flow in urban traffic networks is mainly along arterials, coordination of traffic signals along arterials usually provides numerous advantages [15]. For example, (1) higher level of traffic service is provided in terms of higher overall speed and reduced number of stops; (2) there are few accidents because the platoons of vehicles arrive at each signal when it is green, thereby reducing the possibility of red signal violations and rear-end collisions; (3) greater obedience to the signal commands is obtained from both motorists and pedestrians. The motorists try to keep being within the green interval, and the pedestrian stays at the curb because the vehicles are more tightly spaced. Hence, coordinating the traffic signals along these arterial roads is essential for the effective operation of the urban traffic networks. Some early results can be found in $[6,7]$. In [15], Stamatiadis and Gartner proposed a bandwidth oriented progression model and designed the MULTIBAND96 program, which can produce variable-width progression along arterial road in traffic networks. In [16], Tian and Urbanik reported a signal coordination approach based on a system partition technique to optimize the progression bandwidths for continuous signals along a signalized arterial. Later, Tian et al. [17] developed a computer program which aims to provide maximum bandwidth solutions for randomly generated multiple signal system scenarios. In [18], Kong et al. proposed a bidirection green wave intelligent control technology to solve the coordinate control problems of urban arterial traffic, whereby a two-level control structure was developed. In [19], to optimize the bandwidth for continuous signal along arterial road, Lin et al. developed a new mixedinteger nonlinear programming model with the objection of allowing vehicles to pass through the maximum number of downstream intersections without a stop. In [20], Lu et al. developed an improved two-way bandwidth maximization model for arterial roads with unbalanced bandwidth demands. In their model, a proration impact factor was defined to allow engineers to assign the importance of satisfying the target bandwidth demand rate.

It is noted that all the above progression models and results [16-20] are main focus on arterial signal coordination. It seems that the corresponding results on coordinating urban road networks with green wave coordination strategies are very few. In [21] arterial progression models were first introduced by Gartner and Stamatiadis to deal with the coordination and optimization of traffic signal in urban grid networks. It was shown that the computational efficiency of the optimization model was improved by introducing a heuristic network decomposition procedure. Then, in [22], Gartner and Stamatiadis extended their previous work [21] and developed a network multiband progression model. This model was based on selecting and optimizing an arterial priority network or a route priority network. It should be noted that one of the key aspects of the network decomposition presented in $[21,22]$ was the definition of a priority subnetwork. However, only two simple criteria were presented in $[21,22]$; the details of the selection of a priority subnetwork were not clearly discussed. In addition, coordination control of a large-scale road network should take into account numerous time-varying parameters, such as cycle time, splits, and offset. Although it is technically feasible, it may not always be practical in implementation for real time signal control. It means that a coordination method, which allows simple implementation and nonexcessive data communication, while pointing toward the general direction of alleviating congestion, is needed.

Motivated by the above discussion, in this paper we propose an arterial progression based approach for signal coordination in urban road networks. Simulations on VISSIM have been presented to demonstrate the effectiveness and efficiency of the proposed approach. The main contribution of this paper can be summarized as follows. (1) A bandwidth oriented two-way arterial coordination approach is developed. Different from the typical MAXBAND model [6] and MULTIBAND model [7], one important feature of the proposed method is that not only the phases which provide right of way to the coordinated directions but the phases which provide right of way to uncoordinated directions are also taken into account during the process of optimization of phase sequences; (2) to deal with computational complexity in large-scale road networks, an economical and practical network decomposition strategy is proposed, whereby a novel priority level index is employed to quantify the priority level of each road; (3) the problem of coordinating a large-scale urban road network has been solved by coordinating the principal arterial and several isolated intersections on the boundary of the area, and a novel coordination methodology is proposed.

The reminder of this paper is organized as follows. In Section 2, we propose a new arterial signal coordination model, whereby the phase sequences are optimized. Section 3 presents a novel priority level index and a network decomposition method. A coordination framework of traffic signal control for urban road networks is developed in Section 4. Section 5 reports the results of numerical simulation. Finally, Section 6 concludes this paper with a summary of work.

\section{Coordination of Arterial Road}

Figure 1 illustrates an urban arterial road $A$ that comprises $n$ intersections that are controlled by traffic lights. For each intersection, there are a set of entry and exit links 


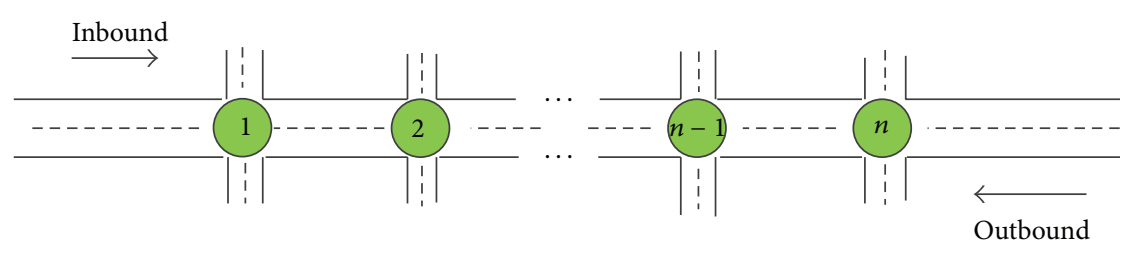

FIgURE 1: An arterial $\operatorname{road} A$ with $n$ intersections.

(approaches), where each link has a fixed length and a set of lanes. For each lane, the "First In and First Out (FIFO)" discipline is used to discharge the vehicle platoons and lane changing or overtaking will not be allowed if vehicles enter a study section [19]. To facilitate the model description, the variables used hereafter are summarized as follows:

(i) $C_{A}$ : the cycle time of all intersections along the arterial $\operatorname{road} A$;

(ii) $g_{j}^{p}$ : the green time of phase $p$ of intersection $j$;

(iii) $l_{j, j+1}$ : the distance between intersection $j$ and intersection $j+1$;

(iv) $v_{j, j+1}$ : the travelling speed between intersection $j$ and intersection $j+1$;

(v) $O_{j, j+1}^{p_{1}}$ : the inbound offset of phase $p_{1}$ between two successive intersections $j$ and $j+1$;

(vi) $O_{j+1, j}^{p_{2}}$ : the outbound offset of coordinated phase $p_{2}$ between two successive intersections $j$ and $j+1$;

(vii) $t_{j}^{p_{1}}$ : the start time of phase $p_{1}$ of intersection $j$ along the inbound direction;

(viii) $t_{j}^{p_{2}}$ : the start time of phase $p_{2}$ of intersection $j$ along the outbound direction;

(ix) $F_{j}$ : the phase set of intersection $j$, that is, $\left\{p_{1}, \ldots, p_{\left|F_{j}\right|}\right\}$.

Figure 2 shows the time space diagram between intersection $j$ and $j+1$. It is shown that, by optimizing the green time of coordinated phase $p_{1}$ and phase $p_{2}$, travel speed $v_{j, j+1}$ and $v_{j+1, j}$, offset $O_{j, j+1}^{p_{1}}$ and $O_{j, j+1}^{p_{2}}$ between intersection, and vehicles along inbound and outbound directions can cross intersection $j$ and $j+1$ without stops simultaneously. The key idea of the proposed arterial road coordination method is to construct a two-way green wave band along two coordinated directions by optimizing and coordinating the green start time of two coordinated phase $p_{1}$ and phase $p_{2}$. Different from previous studies [15-23], in our proposed coordination framework not only the coordinated phase $p_{1}$ and phase $p_{2}$ but the phase $p_{3}$ has been optimized during the process of optimization of phase sequences.

2.1. Cycle Time. First of all, according to the well-known approach known as Websters formula [1], the optimal cycle time of intersection can be expressed as follows:

$$
C_{j}=\frac{1.5 L_{j}+5}{1-\sum_{i \in I_{j}} q_{i} / S_{j}^{i}},
$$

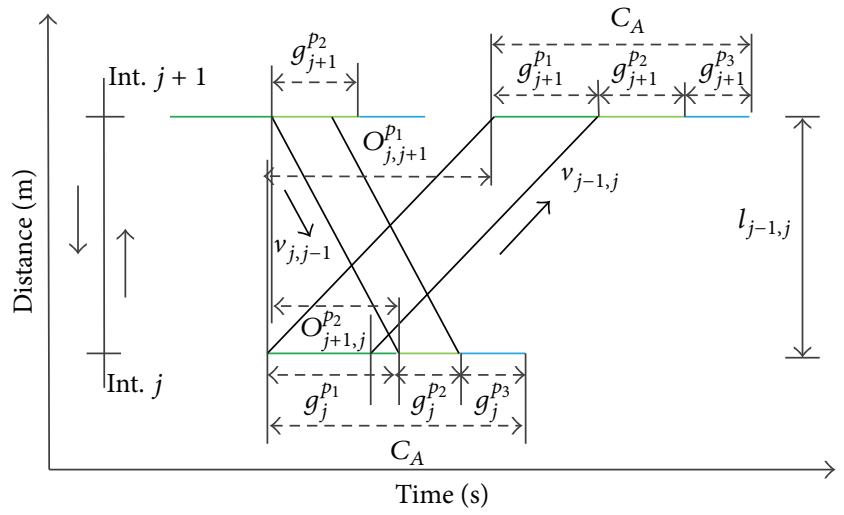

FIGURE 2: The time space diagram between intersections $j-1$ and j.

where $L_{j}$ is lost time of intersection $j, q_{i}$ is the nominal inflow to link $i$ in vehicles per hour, $S_{i}$ is the saturation flow of link $i$, and $I_{j}$ denotes the set of all links which belong to intersection $j$.

In order to facilitate the coordination of an arterial road, each coordinated intersection along the arterial should be associated with an identical cycle time. The intersection with the biggest cycle time is defined as the key intersection of the arterial. The biggest cycle time is usually selected as the common cycle for all intersections along the arterial. For example, the common cycle time $C_{A}$ of the arterial $A$ is defined as

$$
C_{A}=\max _{j \in A} C_{j} .
$$

2.2. Signal Splits. For each phase $p$ of intersection $j$, the optimal green splits can be calculated by the Websters formula [1] as

$$
g_{j}^{p}=\frac{\max \hbar}{\sum_{p \in F_{j}} \max \hbar} \cdot\left(C_{A}-L_{j}\right),
$$

where $\hbar=\left\{q_{j, 1}^{p} / S_{j, 1}^{p}, \ldots, q_{j, i}^{p} / S_{j, i}^{p}, \ldots, q_{j, n}^{p} / S_{j, n}^{p}\right\}, q_{j, i}$ is the inflow to link $i$ of intersection $j, S_{j, i}^{p}$ is the saturation flow of link $i$, and phase $p$ provides the right of way to link $i$.

In general, to guarantee the safety of pedestrians and cyclists, there usually exists a green constraint for each phase $p$ of intersection $j$. Hence, if the green time calculated by (3) is not satisfied by the following constraint:

$$
g_{j, \text { min }}^{p} \leq g_{j}^{p} \leq g_{j, \text { max }}^{p},
$$




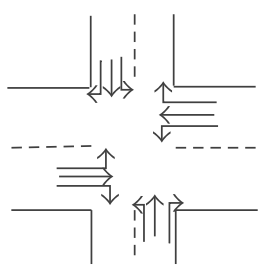

(a)

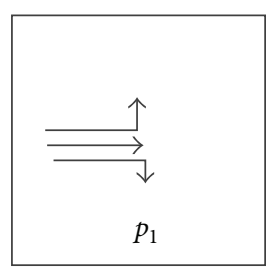

FIGURE 3: (a) Intersection layout, (b) phase setting.
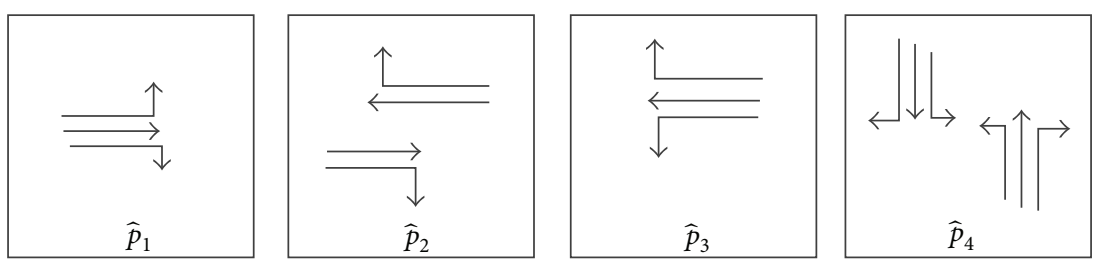

FIgURE 4: Lead/lag left-turn phase.

then we need to regulate $g_{j}^{p}$. Unfortunately, when there are several phases of an intersection which do not satisfy constraint (4), the regulating process will be a tedious work. Therefore, we propose a new method to calculate and regulate the green time $g_{j}^{p}$, as it is shown in the following two equations:

$$
\begin{aligned}
\hat{g}_{j}^{p}=g_{j, \min }^{p} & +\frac{\max \hbar}{\sum_{p \in F_{j}} \max \hbar} \cdot\left(C_{A}-\sum_{p=1} g_{j, \min }^{p}\right), \\
g_{j}^{p} & = \begin{cases}\hat{g}_{j}^{p}, & \text { if } \hat{g}_{j}^{p}<g_{j, \max }^{p}, \\
g_{j, \text { max }}^{p}, & \text { else, }\end{cases}
\end{aligned}
$$

where $g_{j \text {,min }}^{p}$ and $g_{j, \max }^{p}$ are the predefined minimum and maximum green time.

2.3. Phase Setting. As one of the most critical and creative parts of traffic signal coordination, the establishment of a phase scheme has great impact on intersection capacity and efficiency. Note that if left-turn vehicle flows are not controlled, it will severely disturb straight-go vehicles and have big impact on the safety of pedestrians, even if leftturn vehicle flows on arterial road are not heavy [18]. Hence, herein a left-turn phase is designed for the left-turn vehicle flows along arterial road. Consider a typical four-legged intersection shown in Figure 3(a). Regularly, an idyllic phase combination is developed in Figure 3(b) for bidirection arterial signal coordination. It means that phase $p_{2}$ starts just when phase $p_{1}$ completes.

However, in practice to obtain a bidirection green wave band along the arterial road, phase $p_{1}$ and phase $p_{2}$ usually overlap. Assume that phase $p_{1}$ and phase $p_{2}$ provide the right of way for the inbound and outbound traffic flows as shown in Figure 1, respectively. To prevent left-turn traffic
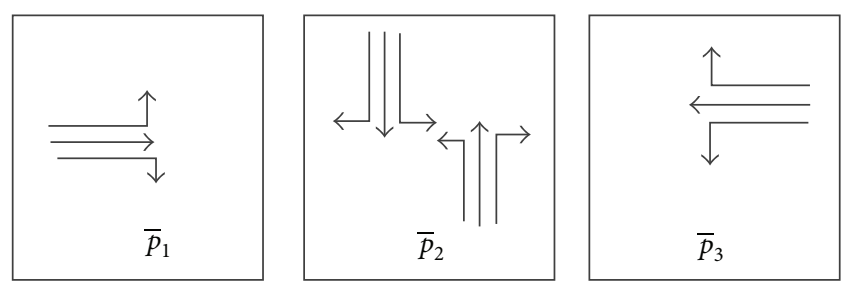

FIGURE 5: Regulated phase sequence.

flows disturbing the straight-go traffic flows, the advanceclose and lag-open strategies are introduced to deal with leftturn phases [18]. Therefore, the phase combination shown in Figure 3(b) can be extended to the phase combination illustrated in Figure 4 . It is clear that the phase $\widehat{p}_{2}$ in Figure 4 is the combination of phase $p_{1}$ and phase $p_{2}$ in Figure 3(b). Herein, the advance-close strategy and the lag-open strategies are applied to the left-turn of phase and that of phase, respectively. Figure 5 shows another phase sequences.

2.4. Speed Constraints. As shown in previous studies [19], to provide smooth traffic flows to users and design adequate signal offsets along an arterial road, the range of link travel time under different traffic conditions should be calculated first. On the other hand, the length of each link is known. Therefore, the following two speed limit constraints (6) are introduced to determine the lower and upper speed limits when the model of maximum progression bandwidth is applied:

$$
\begin{aligned}
& v_{\min } \leq v_{j, j+1} \leq v_{\max } \\
& \bar{v}_{\min } \leq v_{j+1, j} \leq \bar{v}_{\max } .
\end{aligned}
$$


In addition, the following constraints (7) are used to ensure that the speed changes between two adjacent links fall into a reasonable range:

$$
\begin{aligned}
& \widetilde{v}_{\text {min }} \leq\left|v_{j-1, j}-v_{j, j+1}\right| \leq \widetilde{v}_{\text {max }}, \\
& \widehat{v}_{\text {min }} \leq\left|v_{j-1, j}-v_{j, j+1}\right| \leq \widehat{v}_{\text {max }},
\end{aligned}
$$

where $v_{\text {min }}\left(\bar{v}_{\text {min }}, \widetilde{v}_{\text {min }}, \widehat{v}_{\text {min }}\right)$ and $v_{\text {max }}\left(\bar{v}_{\text {max }}, \widetilde{v}_{\text {max }}, \widehat{v}_{\text {max }}\right)$ are predefined constants.

2.5. Calculation of Offsets. The offset between two intersections is defined as the duration from the beginning of a green phase at one intersection to the start of the following nearest green phase at another intersection. In order to obtain a green wave band and allow uninterrupted flows of traffic pass through the entire arterial without stop, the offset between two adjacent intersections can be approximated by the travelling time between the two intersections. Thus, the inbound and outbound offset can be calculated as follows:

$$
\begin{aligned}
& O_{j, j+1}^{p_{1}}=\frac{l_{j, j+1}}{v_{j, j+1}}, \\
& O_{j+1, j}^{p_{2}}=\frac{l_{j, j+1}}{v_{j+1, j}} .
\end{aligned}
$$

2.6. Start Time of Coordination Phases. As discussed above, the phase $p_{1}$ and phase $p_{2}$ shown in Figure $3(\mathrm{~b})$ govern the right of way of the inbound and outbound traffic flow, respectively. Take the arterial road $A$ shown in Figure 1 as an example, assume that the start time of phase $p_{1}$ at intersection 1 along the inbound direction is $t_{0}$; then the start time of phase $p_{1}$ at the $j$ th intersection can be updated by the following equation:

$$
t_{j}^{p_{1}}=t_{0}+\sum_{k=2}^{j} O_{k-1, k}^{p_{1}}, \quad j=2, \ldots, n .
$$

Similarly, if we assume that the start time of phase $p_{2}$ at intersection $n$ along the outbound direction is $\widehat{t}_{0}$, then the start time of phase $p_{2}$ at the $j$ th intersection can be calculated as

$$
t_{j}^{p_{2}}=\widehat{t}_{0}+\sum_{k=j}^{n-1} O_{k+1, k}^{p_{2}}, \quad j=n-1, \ldots, 1
$$

2.7. Objective Function. It is shown in Figure 6(a), corresponding to the phase combination illustrated in Figure 3(b), the ideal case is that when the green time of phase $p_{1}$ (or phase $p_{2}$ ) ends then the green time of phase $p_{2}$ (or phase $p_{1}$ ) starts immediately. However, in practice the length of downstream and upstream road sections is regularly not the same and the travel speed on road sections is heterogeneous. Thus, the ideal case is less likely to happen. There usually exist another two cases. One case shown in Figure 6(b) is that phase $p_{1}$ and phase $p_{2}$ are overlapped. Meanwhile, the phase combination shown in Figure 3(b) is extended to the one illustrated in Figure 4. Another case shown in Figure 6(c) is that, before the start of the green time of phase $p_{2}$ (or phase $p_{1}$ ), the green time of phase $p_{1}$ (or phase $p_{2}$ ) has already ended. In real-world application, Case 3 shown in Figure 6(c) must be avoided.

In [18], to avoid the occurrence of case 3 shown in Figure 6(c) and obtain the maximal progression band, Kong et al. formulated the problem of arterial signal coordination as a biobjective optimization model. In addition, Ye et al. [23] extend the biobjective model to the signal control and coordination for a simple road network constituted by two crossed arterial roads. Since the two objective functions of the biobjective model have different priority level, the problemsolving process is tedious and time-consuming especially for large size problems. Aiming at dealing with this difficultly, we have improved the biobjective model and developed a single objective model. The equation of the objective function is defined as follows:

$$
\min J_{A}=\min \sum_{j=1}^{n}\left|\Delta t_{j}\right|,
$$

subject to (1)-(10) and

$$
\Delta t_{j}= \begin{cases}t_{j}^{p_{2}}-t_{j}^{p_{2}}-g_{j}^{p_{1}}, & \text { if } t_{j}^{p_{1}} \leq t_{j}^{p_{2}} \\ t_{j}^{p_{1}}-t_{j}^{p_{2}}-g_{j}^{p_{2}}, & \text { else. }\end{cases}
$$

Remark 1. Although according to the presented optimization model Case 3 shown in Figure 6(c) only has a small chance of occurring, the occurrence of it can not be completely eliminated. Therefore, to deal with this problem we propose a regulate scheme as a part of model correction.

2.8. Regulate Schemes. Consider Case 2 shown in Figure 6(b); assume that the optimal green time of phase $p_{1}$ and phase $p_{2}$ at the $j$ th intersection is $g_{j}^{p_{1}}$ and $g_{j}^{p_{2}}$, respectively, and the overlapping time is $\Delta t_{j}$. Under this situation, it is naturally to define the overlap interval as a new phase. In other words, the phase setting shown in Figure 3(b) should be extended to that illustrated in Figure 4. It is shown in Figure 7 that the start time and the green time of phase $\widehat{p}_{1}$, phase $\widehat{p}_{2}$, phase $\widehat{p}_{3}$, and phase $\widehat{p}_{4}$ (shown in Figure 4 ) can be derived by (13). For simplicity, herein we assume that $t_{j}^{p_{1}} \leq t_{j}^{p_{2}}$ :

$$
\begin{aligned}
& t_{j}^{\widehat{p}_{1}}=t_{j}^{p_{1}}, t_{j}^{\widehat{p}_{2}}=t_{j}^{p_{2}}, \\
& t_{j}^{\widehat{p}_{3}}=t_{j}^{p_{2}}+g_{j}^{\widehat{p}_{2}}, \quad t_{j}^{\widehat{p}_{4}}=t_{j}^{p_{3}}+g_{j}^{\widehat{p}_{3}},
\end{aligned}
$$



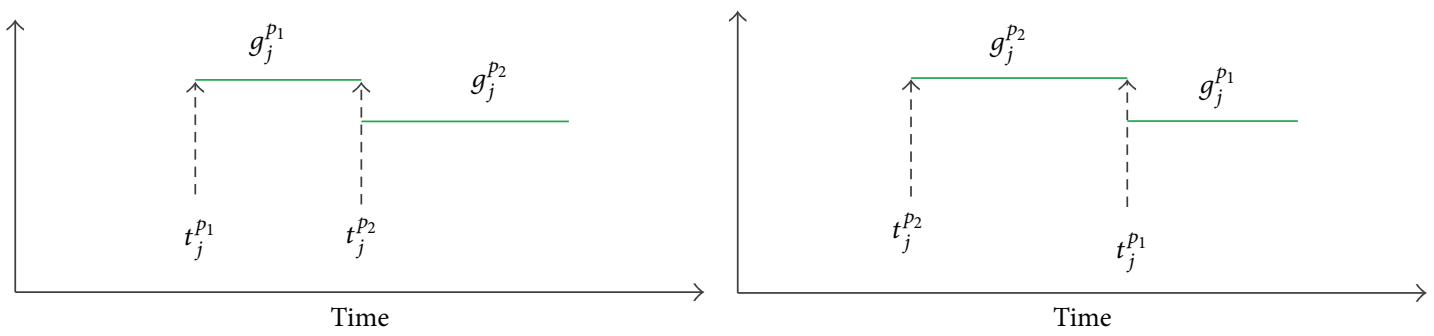

(a) Case 1
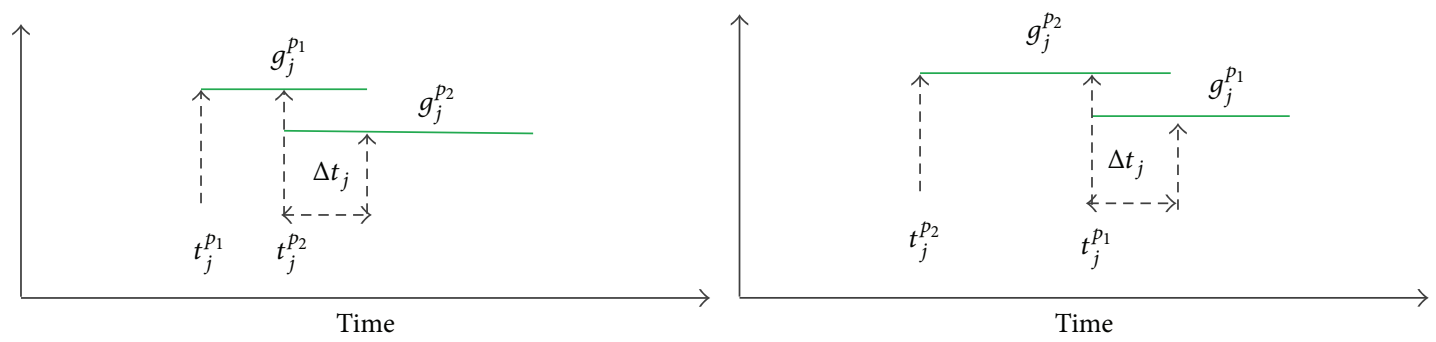

(b) Case 2
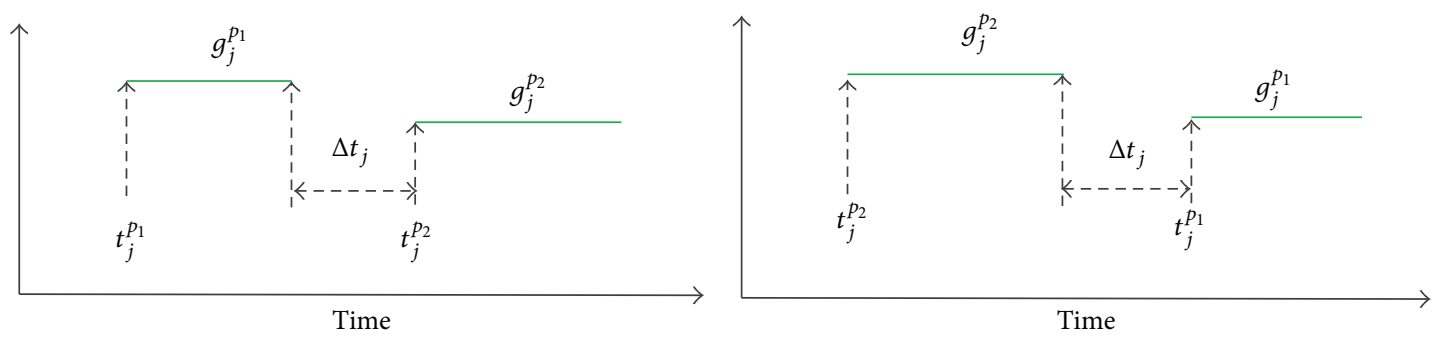

(c) Case 3

FIgURE 6: The relations between the start time of phase $p_{1}$ and phase $p_{2}$.

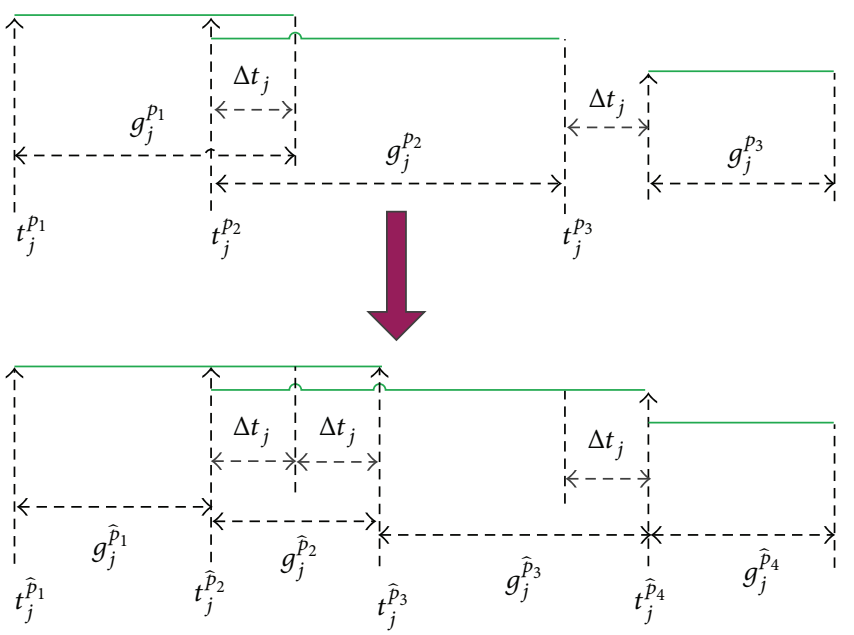

Figure 7: Adjustments for Case 2.

$$
\begin{gathered}
g_{j}^{\widehat{p}_{1}}=g_{j}^{p_{1}}-\Delta t_{j}, \quad g_{j}^{\widehat{p}_{2}}=2 \cdot \Delta t_{j}, \\
g_{j}^{\widehat{p}_{3}}=g_{j}^{p_{2}}, \quad g_{j}^{\widehat{p}_{4}}=g_{j}^{p_{3}} .
\end{gathered}
$$

It should be noted that, for the case $t_{j}^{p_{1}}>t_{j}^{p_{2}}$, similar results can also be derived with the same method. Furthermore, since the length of the cycle time $C_{A}$ is kept unchanged, the overlap time $\Delta t$ should be assigned to phase $\widehat{p}_{1}$ (or $\widehat{p}_{2}$ 
or $\left.\widehat{p}_{3}\right)$. Herein, $\Delta t_{j}$ is assigned to phase $\widehat{p}_{2}$ to maximize the bandwidth.

As mentioned previously, although Case 3 shown in Figure 6(c) should be avoided as much as possible, it might be encountered in real-world traffic conditions. Since the length of overlap time $\Delta t_{j}$ has a significant effect on the length of green time for left-turn traffic of phase $p_{1}$ and that of phase $p_{2}$, we proposed the following three different regulate strategies to respond to the situations that the overlap time $\Delta t$ falls into different ranges.

Subcase $1\left(0 \leq \Delta t_{j}<\delta_{1} \cdot g_{j}^{p_{3}}\right)$. In this scenario, $\Delta t_{j}$ is a small proportion of the green time of phase $p_{3}$. It is argued that one of the simplest ways to eliminate the gap is to prolong the green time of phase $p_{1}$ and advance the green start time of phase $p_{2}$. At the same time, due to the length of the cycle time $C_{A}$ was kept unchanged and the green time of phase $p_{3}$ must be reduced by $\Delta t_{j}$. The result of the regulation is illustrated in Figure 8. Then, we have

$$
\begin{gathered}
\widetilde{t}_{j}^{p_{1}}=t_{j}^{p_{1}}, \quad \widetilde{t}_{j}^{p_{3}}=t_{j}^{p_{3}}, \\
\tilde{t}_{j}^{p_{2}}=t_{j}^{p_{2}}-\frac{\Delta t_{j} \cdot g_{j}^{p_{2}}}{\left(g_{j}^{p_{1}}+g_{j}^{p_{2}}\right)}, \\
\tilde{g}_{j}^{p_{1}}=g_{j}^{p_{1}}+\frac{\Delta t_{j} \cdot g_{j}^{p_{1}}}{\left(g_{j}^{p_{1}}+g_{j}^{p_{2}}\right)}, \\
\tilde{g}_{j}^{p_{2}}=g_{j}^{p_{2}}+\frac{\Delta t_{j} \cdot g_{j}^{p_{2}}}{\left(g_{j}^{p_{1}}+g_{j}^{p_{2}}\right)}, \\
\widetilde{g}_{j}^{p_{3}}=g_{j}^{p_{3}}-\Delta t_{j},
\end{gathered}
$$

where $\delta_{1}$ is a regulator parameter and $\widetilde{t}_{j}^{p_{1}}$ and $\widetilde{t}_{j}^{p_{2}}$ are the start time corresponding to phase $p_{1}$ and phase $p_{2}$ after the regulating strategy is implemented. The phase setting shown in Figure 3(b) is implemented. The start time of phase $p_{1}$ remains unchanged, and that of phase $p_{2}$ would be advanced by $\Delta t_{j} \cdot g_{j}^{p_{2}} /\left(g_{j}^{p_{1}}+g_{j}^{p_{2}}\right)$. In addition, the green time of $p_{1}$ and phase $p_{2}$ is extended, and the sum of them equals the reduction of the green time of phase $p_{3}$.

Subcase $2\left(\delta_{1} \cdot g_{j}^{p_{3}}<\Delta t_{j}<\delta_{2} \cdot g_{j}^{p_{3}}\right)$. Under the current situation, the gap $\Delta t_{j}$ is not a small proportion of the green time of phase $p_{3}$. The approach for eliminating the gap used in previous strategy cannot be adopted. Because if we eliminate the gap by reducing equal amount of green time of phase $p_{3}$, the remaining part of the green time of phase $p_{3}$ will be not sufficient to dissipate all the traffic flows of northbound and southbound at intersection $j$ in one cycle. As a result, extra queuing delay will be imposed on part of the traffic flows of the minor streets whose right of way is controlled by phase $p_{3}$.

Herein, to eliminate the gap $\Delta t_{j}$, on one hand we advance the start time of phase $p_{1}$ and that of phase $p_{2}$. Simultaneously, to reduce the influences of these regulations imposed on coordinated directions, we also regulate the length of

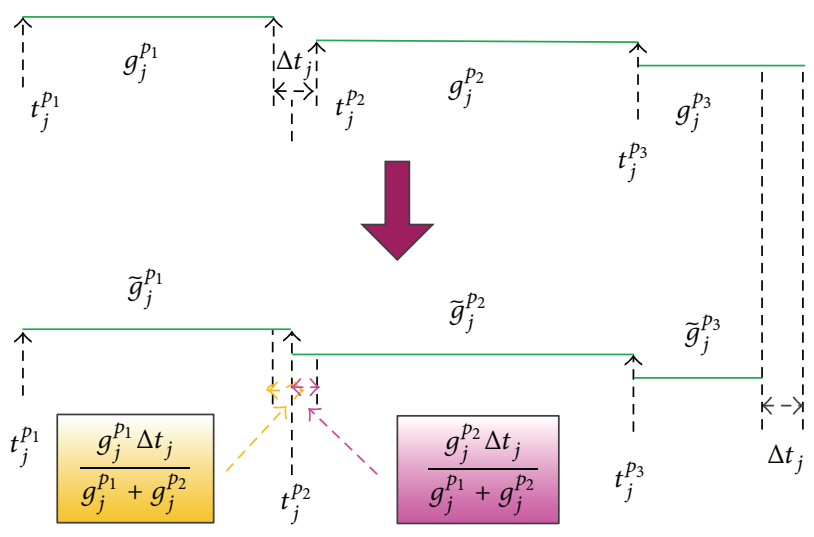

Figure 8: Adjustments for Subcase 1.

the green time of phase $p_{1}$ and that of phase $p_{2}$. As shown in Figure 9, the start time and green time of phase $p_{1}$, phase $p_{2}$, and phase $p_{3}$ are calculated as

$$
\begin{gathered}
\widetilde{t}_{j}^{p_{1}}=t_{j}^{p_{1}}+\frac{\Delta t_{j} \cdot g_{j}^{p_{1}}}{\left(g_{j}^{p_{1}}+g_{j}^{p_{2}}+g_{j}^{p_{3}}\right)}, \\
\widetilde{t}_{j}^{p_{2}}=t_{j}^{p_{2}}-\frac{\Delta t_{j} \cdot g_{j}^{p_{2}} \cdot g_{j}^{p_{3}}}{\left(g_{j}^{p_{2}}+g_{j}^{p_{2}}\right)\left(g_{j}^{p_{2}}+g_{j}^{p_{2}}+g_{j}^{p_{3}}\right)} \\
-\frac{\Delta t_{j} \cdot g_{j}^{p_{2}}}{\left(g_{j}^{p_{2}}+g_{j}^{p_{2}}+g_{j}^{p_{3}}\right)}, \\
\widetilde{t}_{j}^{p_{3}}=t_{j}^{p_{3}}-\frac{\Delta t_{j} \cdot g_{j}^{p_{2}}}{\left(g_{j}^{p_{2}}+g_{j}^{p_{2}}+g_{j}^{p_{3}}\right)}, \\
\widetilde{g}_{j}^{p_{1}}=g_{j}^{p_{1}}+\frac{\Delta t_{j} \cdot g_{j}^{p_{1}} \cdot g_{j}^{p_{3}}}{\left(g_{j}^{p_{2}}+g_{j}^{p_{2}}\right)\left(g_{j}^{p_{2}}+g_{j}^{p_{2}}+g_{j}^{p_{3}}\right)}, \\
\widetilde{g}_{j}^{p_{2}}=g_{j}^{p_{2}}+\frac{\Delta t_{j} \cdot g_{j}^{p_{2}} \cdot g_{j}^{p_{3}}}{\left(g_{j}^{p_{2}}+g_{j}^{p_{2}}\right)\left(g_{j}^{p_{2}}+g_{j}^{p_{2}}+g_{j}^{p_{3}}\right)}, \\
\widetilde{g}_{j}^{p_{3}}=g_{j}^{p_{3}}-\frac{\Delta t_{j} \cdot g_{j}^{p_{3}}}{\left(g_{j}^{p_{1}}+g_{j}^{p_{2}}+g_{j}^{p_{3}}\right)},
\end{gathered}
$$

where $\delta_{2}$ is a regulator parameter. The phase setting shown in Figure 3(b) is implemented.

Subcase $3\left(\delta_{2} \cdot g_{j}^{p_{3}}<\Delta t_{j}<g_{j}^{p_{3}}\right)$. Different from the previous subcase 1 and subcase 2, for the current condition the gap $\Delta t_{j}$ is close to the length of green time of phase $p_{3}$. In other words, compared with the length of green time of phase $p_{1}$ and phase $p_{2}, g_{j}^{p_{3}}-\Delta t_{j}$ is small. As a result, phase $p_{3}$ can be inserted between phase $p_{1}$ and phase $p_{2}$ to eliminate the gap. Meanwhile, the start time of phase $p_{1}$ should be advanced, and the start time of phase should be lagged. As illustrated in 


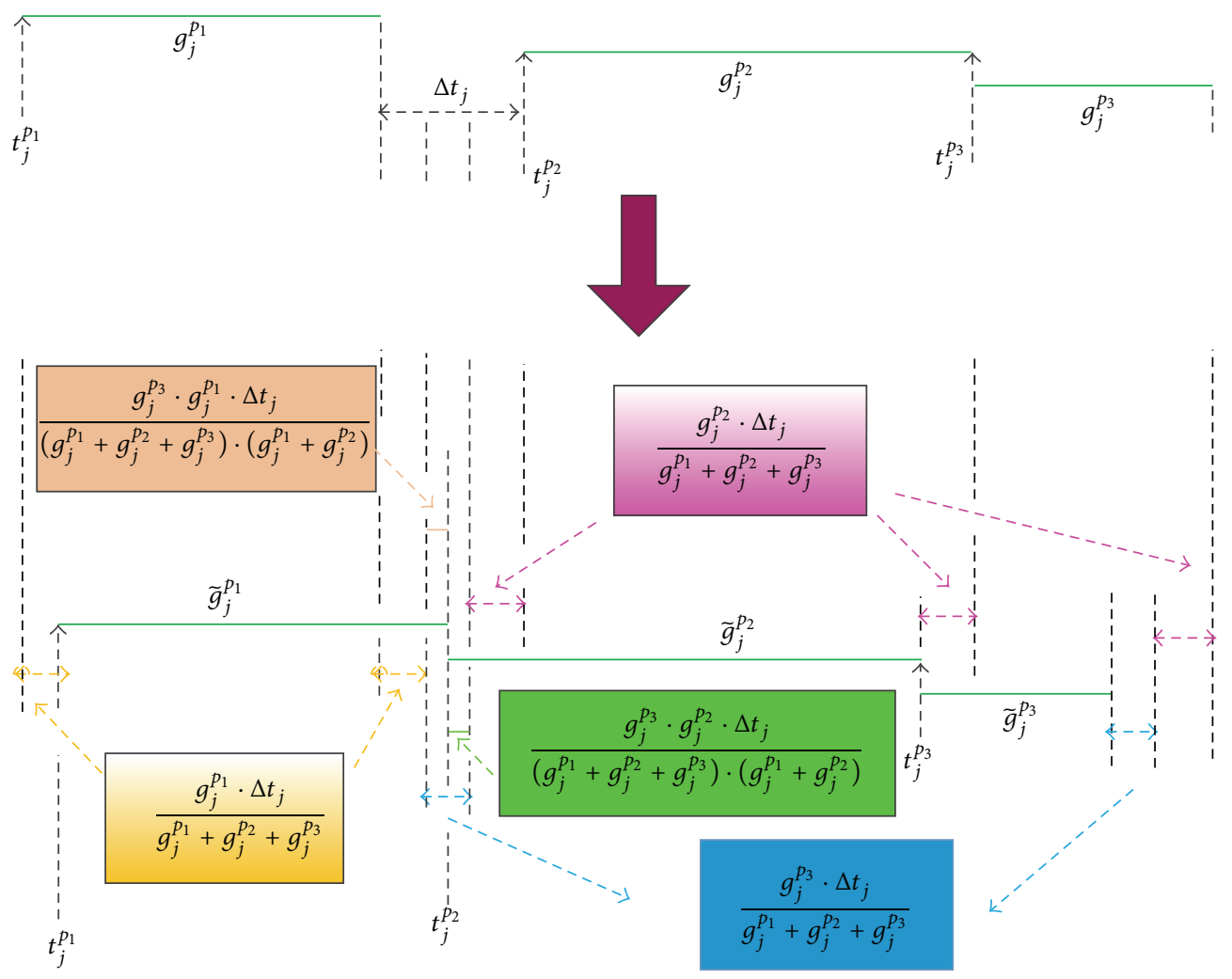

Figure 9: Adjustments for Subcase 2.

Figure 10, the start time and the length of green time can be regulated with the following equations:

$$
\begin{gathered}
\bar{p}_{j}^{\bar{p}_{1}}=t_{j}^{p_{1}}-\frac{\left(g_{j}^{p_{3}}-\Delta t_{j}\right) \cdot g_{j}^{p_{1}}}{\left(g_{j}^{p_{1}}+g_{j}^{p_{2}}\right)}, \\
t_{j}^{\bar{p}_{2}}=t_{j}^{\bar{p}_{1}}+g_{j}^{\bar{p}_{1}}, \quad t_{j}^{\bar{p}_{3}}=t_{j}^{\bar{p}_{2}}+g_{j}^{\bar{p}_{2}}, \\
g_{j}^{\bar{p}_{1}}=g_{j}^{p_{1}}, \quad g_{j}^{\bar{p}_{2}}=g_{j}^{p_{2}}, \\
g_{j}^{\bar{p}_{3}}=g_{j}^{p_{3}} .
\end{gathered}
$$

Under this condition, the phase sequence defined in Figure 3(b) is extended as a new one shown in Figure 5. Compared with the phase setting in Figure 3(b), the phase which provides right of way for minor roads is in advance.

Remark 2. On one hand, the common cycle time $C_{A}$ of all intersections on the arterial $\operatorname{road} A$ is kept unchanged during the coordination process. On the other hand, phase $p_{1}$, phase $p_{2}$, and phase $p_{3}$ are periodically implemented. Therefore, the gap $\Delta t_{j}$ between phase $p_{1}$ and phase $p_{2}$ is always limited in the interval $\left[k_{1} \cdot C_{A}, k_{2} \cdot C_{A}+g_{j}^{p_{3}}\right], k_{1}, k_{2} \in \mathbb{Z}^{+}$. As a result, herein only the upper three conditions were discussed.

\section{Network Decomposition}

On one hand, decomposition of system structures or models is a prerequisite in coordination and control of large-scale complex systems. On the other hand, as a large-scale complex system, urban traffic system is usually composed by numerous interconnected subsystems. It is difficult to be controlled with centralized control structure due to the high computational burden, communication bandwidth limitations, and robustness and reliability problem [26]. Therefore, many decomposition strategies have been proposed in previous literatures $[16,22,24,25]$.

In [16], Tian and Urbanik proposed a system partition technology for signal timing of large signalized arterial, whereby the decomposition strategy is purely heuristic and defines three to five intersections as a subsystem. In [24], Zhou and $\mathrm{Lu}$ defined each intersection and its connected roads as a subsystem and developed a neighbor-based distributed coordination scheme to enhance the computational efficiency. In [22], Gartner and Stamatiadis proposed an iterative heuristic network decomposition framework, in which a priority subnetwork would be defined firstly in each iteration. The priority network was chosen so that it contained only a tree of arterials, eliminating any network loops. However, Gartner et al. only presented two simple criteria to select a priority subnetwork, without details of the operation and definition of a priority subnetwork. For this reason, in this paper we define a priority level index first and then develop a novel netowork decomposition approach. 


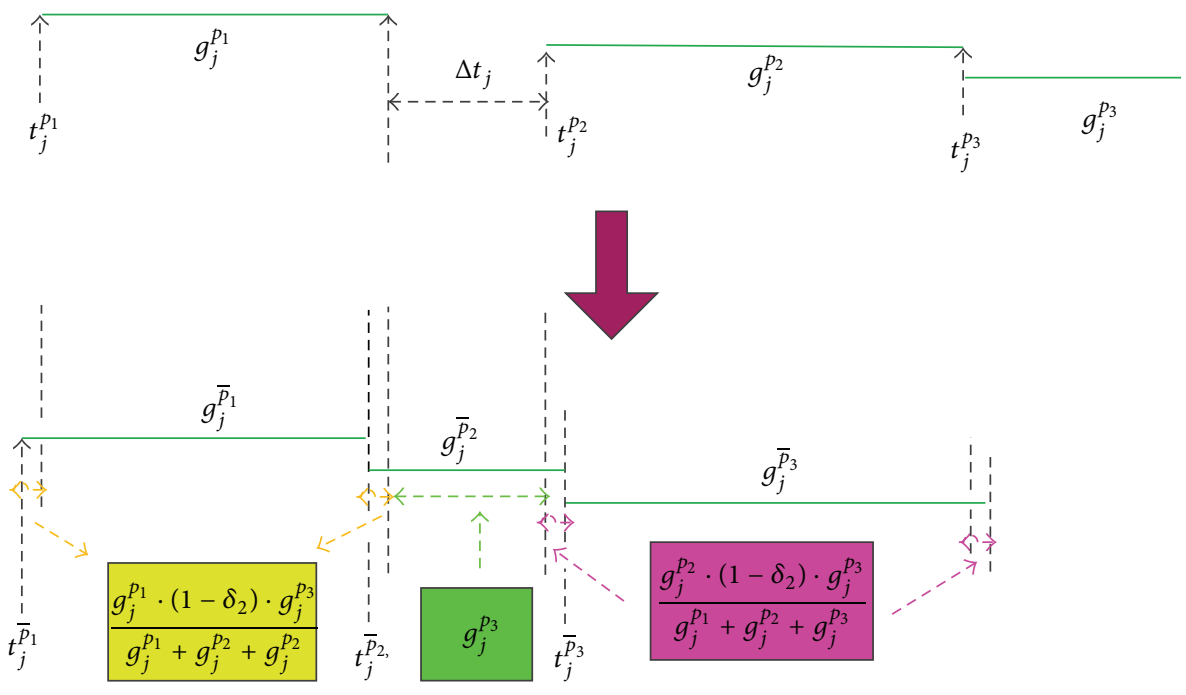

Figure 10: Adjustments for Subcase 3.

3.1. Priority Level Index. In order to quantify the priority level of each road (including arterial and minor roads) in road networks, we develop a priority level index which is defined as

$$
\begin{gathered}
\mathrm{PL}_{A}=w_{1} * X_{A}+w_{2} * Q_{A}+w_{3} * E_{A} \\
w_{1}+w_{2}+w_{3}=1
\end{gathered}
$$

where $\mathrm{PL}_{A}$ denotes the value of the priority level index of road $A$ and $X_{A}$ and $Q_{A}$ are the saturation degree and throughput of road $A$, respectively. $E_{A}$ is the score decided by experts or engineers. $w_{1}, w_{2}$, and $w_{3}$ are the weights.

The saturation $X_{A}$ of road $i$ is calculated by

$$
X_{A}=\frac{1}{n_{A}} \cdot \sum_{j \in A} \sum_{p \in F_{j}} \max _{i \in I_{j}^{p}}\left\{\frac{q_{j, i}^{p}}{g_{j, i}^{p} \cdot S_{j, i}}\right\},
$$

where $F_{j}$ is the phase set of intersection $j, q_{j, i}^{p}$ and $S_{j, i}$ are the nominal inflow and saturation flow of link $i$ in vehicles per hour, respectively, and $n_{A}$ is the number of intersections on arterial $\operatorname{road} A$.

The throughput of road $A$ is calculated by

$$
Q_{A}=\sum_{j \in A} \sum_{p \in F_{j}^{c o}} q_{j}^{p},
$$

where $F_{j}^{c o}$ is the phase set which governs the right of way of the traffic flow along the inbound and outbound directions and $q_{j}^{p}$ is the traffic flow corresponding to phase $p$ of intersection $j$.

Instead of designing a heuristic scheme, herein we introduce a score index $E_{A}$ to quantify the expert knowledge. Furthermore, based on the specific network topology and the history volumes of traffic flow of each road, the score $E_{A}$ is estimated by experts or engineers.
Remark 3. Note that when we use (17) to calculate the priority level index $\mathrm{PL}_{A}, X_{A}, Q_{A}$, and $E_{A}$ should be normalized into the interval $[0,1]$ first. In addition, since the proposed method is a fixed-time control strategy and the traffic flows in road networks can be assumed to be steady-state, the vehicular traffic flow data used in (19)-(20) can be estimated by history traffic data collected by inductive loop detectors or magnetic sensors.

3.2. Decomposition Algorithm. The decomposition algorithm described herein does not merely use some heuristic schemes, as most previous method do, but rather it is based on the priority level index. Central to our decomposition algorithm is to order the principle arterial roads of the network according to the priority index. The arterial roads with higher priority index PL are picked out firstly, and then a threshold $\tilde{n}=\gamma \cdot n$ is set to guarantee that the key intersections insider the network will be selected. It should be noted that the parameter $\gamma \in(0,1]$ can be dynamically regulated according to the coordination performance of the road networks. Finally, we summarized the network decomposition approach in the following.

\section{Algorithm 4}

Step 1. Collect the topology information and the traffic flow characteristics of the studied road network. Set $R$ as an empty set and the iteration index $\ell=1$.

Step 2. Calculate the priority level of each arterial (or minor) road with (17), sort them in decreasing order, and store them in a set $B$.

Step 3. Pick out the road $A$ with the highest priority level in the set $B-R$ and place it into the set $C$. 
Step 4. Count the number of intersections that belong to the roads of set $C$ and mark it as $n^{c o}$; denote the total number of intersections in the network as $n$.

Step 5. Update the result set $R=B(1: \ell)$ that is a set constituted by the first $\ell$ elements in the set $B$.

Step 6. Check if the termination iteration condition $n^{c o} \geq \gamma \cdot n$ is satisfied. If the termination condition is satisfied, then end the iteration and return $R$.

Step 7. Let $\ell=\ell+1$; go back to Step 3 .

\section{Coordination of Urban Traffic Networks}

It is common knowledge that the predominant traffic flows in urban traffic networks are along arterial roads. Coordination of traffic signals along arterial roads offers numerous benefits as indicated in previous literatures [15-23]. Therefore, coordinating the traffic signals along these arterial roads is essential for the effective operation of the urban traffic networks. In this section, we propose a novel coordination approach for urban traffic networks, which is abbreviated as CoA in the remainder of this paper. The basic idea of our coordination approach is that the problem of coordinating an urban traffic network can be transformed into that of coordinating the principal arterial roads and scattered isolated intersections on the boundary of the road networks.

In general, based on the coordination model of an arterial road, the objective function of an urban traffic network can be defined as

$$
\min \sum_{A \in N} J_{A}=\min \sum_{A \in N} \sum_{j=1}^{n_{A}}\left|\Delta t_{j}\right|,
$$

subject to the constraints (1)-(10) and (12), where $N$ denotes a traffic network. It indicates that the objective function is to maximize the width of the green wave band of the whole network which includes not only the arterial roads but the minor roads.

However, in real-life traffic conditions and applications, the minor roads are not suitable to be coordinated with green wave coordination technology due to lower traffic demands and physical topology of minor roads. In addition, traffic flows are mainly concentrated in principle arterial roads. Hence, herein based on the network decomposition approach proposed in previous section, we define an objective function as follows:

$$
\min \sum_{A \in R} \omega_{A} \cdot J_{A}=\min \sum_{A \in R} \omega_{A} \cdot \sum_{j=1}^{n_{A}}\left|\Delta t_{j}\right|,
$$

where $\omega_{A}$ is the weight coefficient which reflects the priority level of arterial road $A$ and $R$ is the road set with higher priority level and is calculated by Algorithm 4. It means that only the intersections which belong to the key arterial roads set are coordinated.

In addition, it should be noted that if an intersection belongs to two coordinate arterials, it means that there

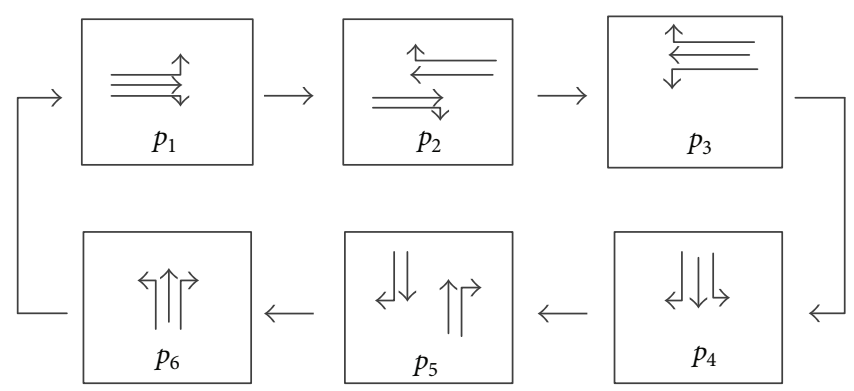

FIgURE 11: A phase sequence for two-crossed arterial roads.

are two major coordination directions. Therefore, the phase setting shown in Figure 4 should be regulated. As shown in Figure 11, another phase setting is presented. For example, intersection 6 and intersection 12 (shown in Figure 12) have six phases.

Based on the arterial signal coordination and network decomposition approach presented in previous sections, our proposed coordination approach CoA for the whole road network is described in Algorithm 6.

Remark 5. As shown in Figure 7, the grid network consisted of eight arterial (or minor) roads and fifteen intersections. Assume that according to (17) we have the set $B=$ $\left\{A_{1}, A_{2}, \ldots, A_{8}\right\}$, which implies that arterial road $A_{1}$ has the highest priority level while $A_{8}$ has the smallest priority level. Setting $\gamma=0.65$, then utilizing Algorithm 4, we can get the decomposition result set $B=\left\{A_{1}, A_{2}, A_{3}\right\}$. It means that only the three principal arterial roads are coordinated, while the remaining intersections $1,4,9,12$, and 13 are controlled by fixed-time control strategy.

\section{Algorithm 6.}

Step 1 (Initialization). Based on the network decomposition Algorithm 4 a given traffic road network can be decomposed into a set of arterials $R$. Each arterial can be seen as a subnetwork which contains the bulk traffic flows of the network. Coordinate the signals along the arterial with the highest priority level in $R$ that is $R(1)$ with the proposed arterial coordination approach in Section 2; then let $\ell=2$.

Step 2 (check). If arterial $R(\ell)$ will form a loop with the previous coordinated arterials, then go to Step 4; else go to Step 3.

Step 3 (coordination). Coordinate the arterial $R(\ell)$ with the proposed arterial coordination approach in Section 2.

Step 4 (loop). If $\ell<|R|$, let $\ell=\ell+1$ and go back to Step 2; else terminate the iteration.

Step 5 (complementary). Fixed-time control strategy or actuated control strategy is implemented to the intersections of the network except those along the coordinated arterials.

Step 6 (finish). Stop the coordination process. 


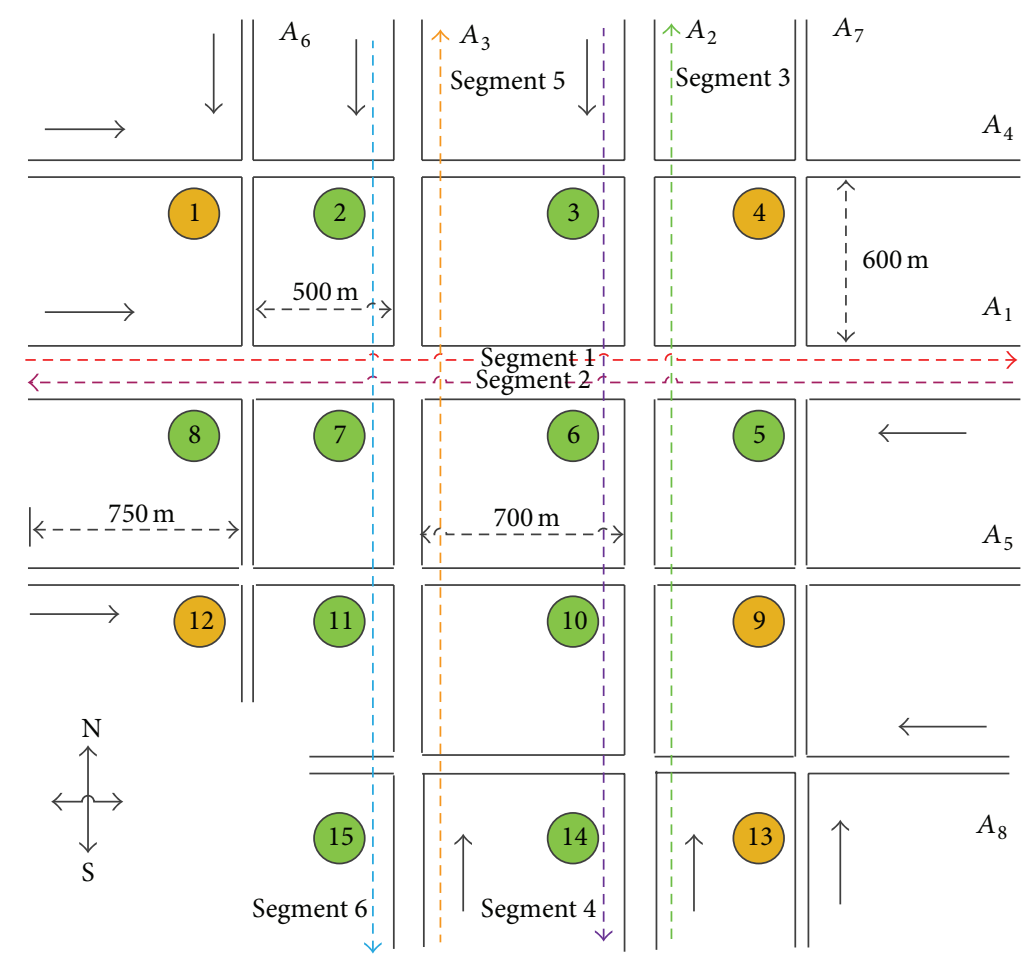

FIGURE 12: Simulation road network.

\section{Simulation Results}

In this section, to demonstrate the effectiveness and efficiency of the proposed signal coordination approach, measures of effectiveness (MOE) acquired by VISSIM, including average delay, average travel time, average queue length, and stops per vehicle on each travel segment, are introduced.

The simulation network is created as shown in Figure 12, and the road network includes eight arterial roads and fifteen signalized intersections. $A_{1}$ is a bidirection arterial road with six lanes and $A_{2}$ and $A_{3}$ are bidirection arterial road with four lanes. One direction and one lane are defined for the other roads. The width of each lane is 3.5 meters. As marked in Figure 12, to evaluate the coordination performance, we define six travel segments, which undertake the main traffic demand of the road network. The input flow and tuning rates of the road network are presented in Tables 1 and 2 , respectively. We assume the average vehicle length is 5 meters, and the minimum and maximum free vehicle speed is $40 \mathrm{~km} / \mathrm{h}$ and $50 \mathrm{~km} / \mathrm{h}$, respectively.

Moreover, as we choose the default traffic configuration, which contains $98 \%$ cars and $2 \%$ heavy good cars, herein the saturated rate of each link is set as $2000 \mathrm{veh} / \mathrm{h}$ (refer to the manual of Vissim 3.7). All other parameters, such as lanechange distance, minimum headway, and waiting time before diffusion, are using the default settings.

The proposed model is simulated by VISSIM 3.7. A fixedtime control (FC) strategy is used as a reference to the proposed coordination ( $\mathrm{CoA})$ method. In order to evaluate the performance of CoA under different traffic demands, the simulations are implemented under three scenarios, according to different input traffic flow rates supplied to the network as shown in Table 2. Firstly, the simulation results are compared for these different scenarios. The average travel time along each segment is shown in Figure 13. According to it, we can clearly see that CoA performs better than that of FC on all travel segments under different scenarios, and the average travel time is reduced by around $9.27 \%-11.68 \%$. The travel time of segments 1,3 , and 5 is much bigger than that of segments 1,3 , and 5 . This is mainly due to the input traffic flows of segments 1,3 , and 5 which are high and the traffic condition is more crowded.

Figure 14 illustrates the delay time per vehicle on each travel segment under different traffic demands. Compared with FC, the average delay time of CoA is reduced by about $27.5 \%-33.81 \%$. Moreover, with the increase of traffic demand, the rate of decrease of delay time is greater. Under the FC control strategy, for Case 1 and Case 2 the maximum delay time is 140 (s/veh), while it is increased quickly to 260 (s/veh) for Case 3. However, with the proposed control strategy CoA, the increase of delay time under different traffic demand is relatively flat. It illustrates that the robustness of CoA is better than that of FC.

Figure 15 shows that the average number of stops per vehicle on each travel segment increases remarkably with the traffic demand increase. It can be seen that CoA achieves better performance than that of FC under all traffic scenarios. Compared with the FC strategy, CoA obtains much less stops (per vehicle), particularly for Case 3 which has much higher traffic demand. The reason is that with bidirection green 
TABLE 1: Turning rates.

\begin{tabular}{|c|c|c|c|c|c|c|c|c|c|c|c|c|}
\hline \multirow{2}{*}{ Int. } & \multicolumn{3}{|c|}{ Northbound } & \multicolumn{3}{|c|}{ Westbound } & \multicolumn{3}{|c|}{ Southbound } & \multicolumn{3}{|c|}{ Eastbound } \\
\hline & LT & $\mathrm{TH}$ & RT & LT & $\mathrm{TH}$ & RT & LT & $\mathrm{TH}$ & RT & LT & $\mathrm{TH}$ & RT \\
\hline 1 & & 0.8 & 0.2 & & & & 0.15 & 0.85 & & & & \\
\hline 2 & 0.15 & 0.75 & 0.1 & & & & 0.15 & 0.85 & & & 0.8 & 0.2 \\
\hline 3 & 0.15 & 0.7 & 0.15 & & & & 0.15 & 0.85 & & & 0.85 & 0.15 \\
\hline 4 & 0.15 & 0.85 & & & & & & & & & 0.7 & 0.3 \\
\hline 5 & 0.1 & 0.9 & & & 0.87 & 0.13 & & & & 0.05 & 0.75 & 0.2 \\
\hline 6 & 0.07 & 0.83 & 0.10 & 0.07 & 0.81 & 0.12 & 0.05 & 0.79 & 0.16 & 0.09 & 0.73 & 0.18 \\
\hline 7 & 0.05 & 0.85 & 0.10 & 0.05 & 0.84 & 0.11 & 0.05 & 0.80 & 0.15 & 0.05 & 0.76 & 0.19 \\
\hline 8 & & 0.9 & 0.1 & 0.08 & 0.92 & & 0.2 & 0.7 & 0.1 & & & \\
\hline 9 & 0.2 & 0.8 & & & & & & & & & 0.8 & 0.2 \\
\hline 10 & 0.06 & 0.84 & 0.1 & & & & 0.1 & 0.9 & & & 0.83 & 0.17 \\
\hline 11 & 0.05 & 0.83 & 0.12 & & & & 0.11 & 0.89 & & & 0.85 & 0.15 \\
\hline 12 & & 0.8 & 0.2 & & & & 0.08 & 0.92 & & & & \\
\hline 13 & & & & & 0.9 & 0.1 & & & & 0.1 & 0.9 & \\
\hline 14 & & & & 0.18 & 0.75 & 0.07 & & 0.9 & 0.1 & 0.05 & 0.95 & \\
\hline 15 & & & & 0.15 & 0.8 & 0.05 & & 0.85 & 0.15 & 0.07 & 0.93 & \\
\hline
\end{tabular}

TABLE 2: Input traffic flow (veh/h).

\begin{tabular}{|c|c|c|c|c|c|c|c|c|c|}
\hline \multirow{2}{*}{ Scenarios } & \multirow{2}{*}{ Entrance } & \multicolumn{8}{|c|}{ Input traffic flow of each arterial road } \\
\hline & & $A_{1}$ & $A_{2}$ & $A_{3}$ & $A_{4}$ & $A_{5}$ & $A_{6}$ & $A_{7}$ & $A_{8}$ \\
\hline \multirow{4}{*}{ Case 1} & Eastbound & 1200 & & & 300 & 500 & & & \\
\hline & Westbound & 1000 & & & & & & & 300 \\
\hline & Southbound & & 800 & 900 & & & 350 & & \\
\hline & Northbound & & 1000 & 950 & & & & 300 & \\
\hline \multirow{4}{*}{ Case 2} & Eastbound & 1800 & & & 400 & 450 & & & \\
\hline & Westbound & 1500 & & & & & & & 350 \\
\hline & Southbound & & 1200 & 1350 & & & 400 & & \\
\hline & Northbound & & 1500 & 1450 & & & & 400 & \\
\hline \multirow{4}{*}{ Case 3} & Eastbound & 2400 & & & 450 & 500 & & & \\
\hline & Westbound & 2000 & & & & & & & 400 \\
\hline & Southbound & & 1500 & 1600 & & & 450 & & \\
\hline & Northbound & & 1800 & 1750 & & & & 450 & \\
\hline
\end{tabular}

wave coordination the traffic flows along arterial roads are smoothed. Many vehicles can cross two or more adjacent intersections without stops.

The average queue length on each travel segment is reported in Figure 16; we can find that there are a few queued vehicles for both control strategies CoA and FC under Case 1 which has the lowest traffic demand. However, as the traffic demand increases from Case 1 to Case 3, the queue length grows quickly. In comparison with $\mathrm{FC}$, the average queue length of CoA is greatly reduced.

Another observation on the performance curves of queue length shown in Figure 11 is that the queue length of the travel segments 1,3 , and 5 is much bigger than that of 2, 4, and 6 . This is mainly due to the input traffic flows for segments 1,3 , and 5 are bigger than that of the remaining three segments. As shown in Figure 13 to Figure 16, it should be noted that CoA performs much better on segments 1,3 , and 5 than that of on segments 2, 4, and 6 . The reason for this phenomenon is that, during the process of regulating the start time of inbound and outbound, we prefer to guarantee the coordination efficiency of the direction with higher traffic demand.

The performance index of the whole network is presented in Table 3 and Figure 17. According to it, we can find that the proposed method CoA performance is much better than that of FC. In particularly, compared with FC, the average delay time, stops per vehicle, and average queue length are greatly reduced at least by about $27.5 \%-33.88 \%$ under different traffic scenarios. 


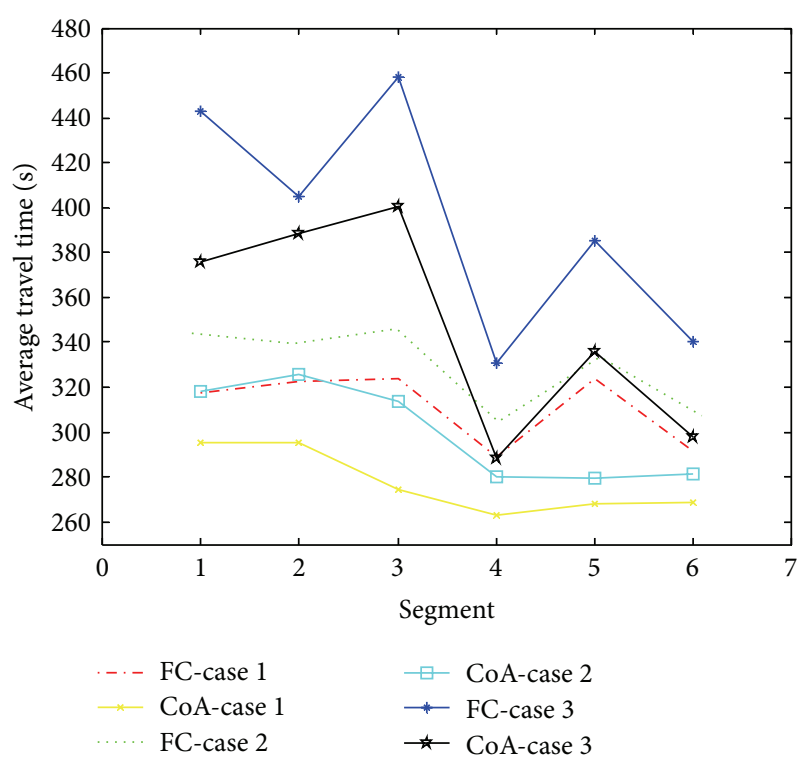

FIGURE 13: Comparison of average travel time on different travel segment.

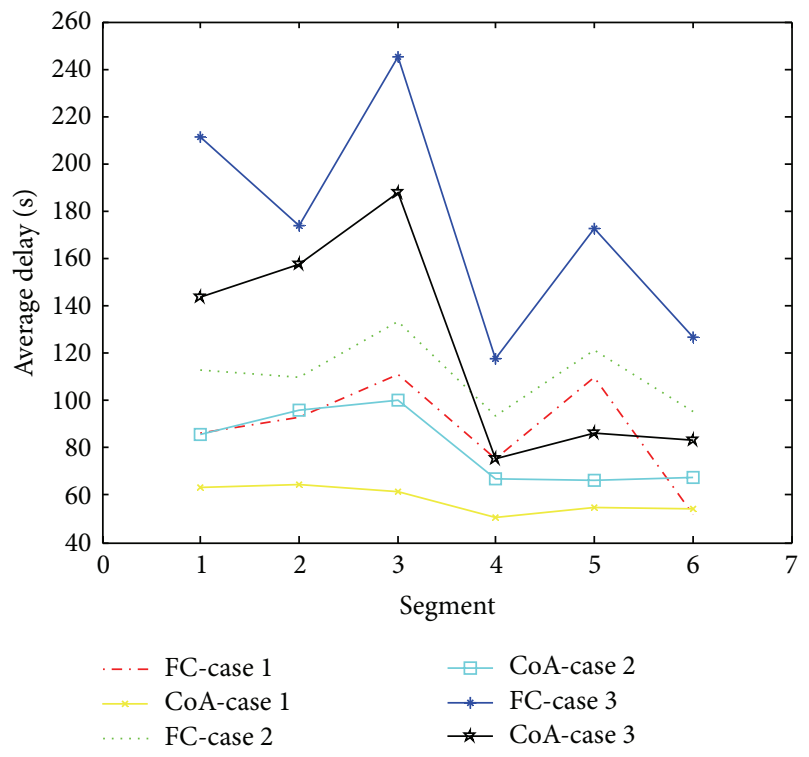

FIGURE 14: Comparison of average delay time on different travel segment.

\section{Conclusion}

This paper investigates the optimization and coordination of signal splits in large-scale urban road networks. To reduce the high computational complexity and improve the applicability of signal coordination methods, a novel method based on a system decomposition strategy was proposed. The key idea of the proposed method is that a large-scale network coordination problem has been solved by coordinating several principle arterial roads and several isolated intersections on the boundary of the area. In this study, to satisfy the minimum (maximum) green time constraints, which are used to

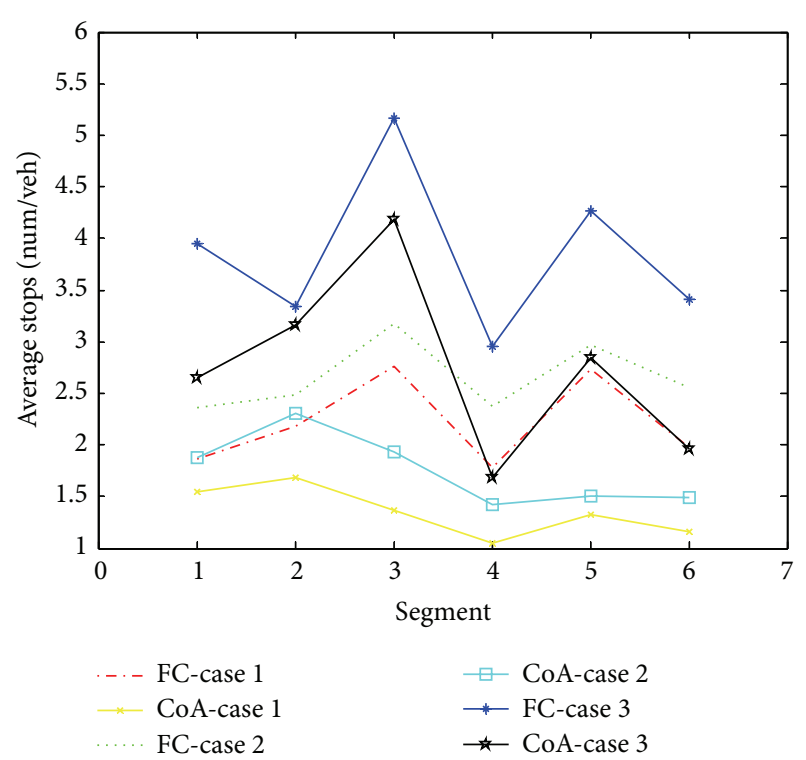

FIGURE 15: Comparison of average stops per vehicle on different travel segment.

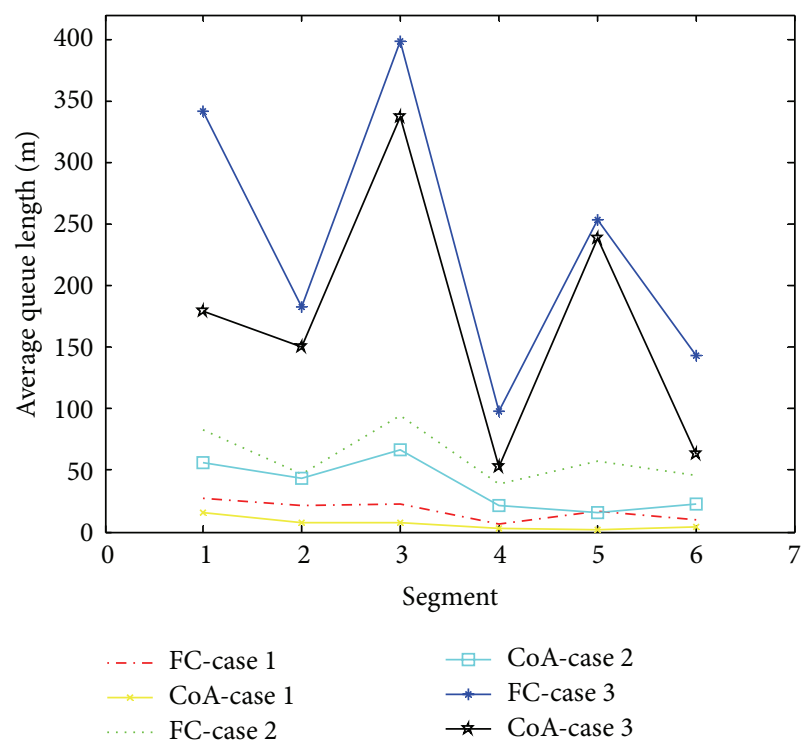

FIGURE 16: Comparison of average queue length on different travel segment.

guarantee the safety of pedestrians and cyclists, we propose a strategy to regulate the calculated green time. In addition, a new two-way arterial signal coordination model is developed. Different from previous studies, not only the phases which provide right of way to the coordinated directions but also the phases which provide right of way to uncoordinated directions were optimized in our proposed arterial coordination method. Moreover, a system decomposition strategy was reported, whereby a novel priority level index is defined to quantify the priority level of each road. The performance of the proposed method CoA has been validated by VISSIM under various traffic conditions. Simulation results show that 
TABLE 3: Measurements of effectiveness.

\begin{tabular}{|c|c|c|c|c|c|}
\hline Scenarios & Control strategy & Travel time (s) & Delay (s/veh) & Stops (num/veh) & Queue (m) \\
\hline \multirow{2}{*}{ Case 1} & $\mathrm{FC}$ & 1867.7 & 527.3 & 13.29 & 104 \\
\hline & $\mathrm{CoA}$ & 1664.2 & 349 & 8.14 & 39 \\
\hline \multicolumn{2}{|c|}{ Improved } & $10.9 \%$ & $33.81 \%$ & $38.75 \%$ & $62.5 \%$ \\
\hline \multirow{3}{*}{ Case 2} & FC & 1981.8 & 666.2 & 15.94 & 366 \\
\hline & $\mathrm{CoA}$ & 1798 & 483 & 10.54 & 227 \\
\hline & ved & $9.27 \%$ & $27.5 \%$ & $33.88 \%$ & $37.98 \%$ \\
\hline \multirow{3}{*}{ Case 3} & FC & 2362.5 & 1047.5 & 23.1 & 1419 \\
\hline & $\mathrm{CoA}$ & 2086.6 & 733.9 & 16.48 & 1020 \\
\hline & ved & $11.68 \%$ & $29.94 \%$ & $28.66 \%$ & $28.12 \%$ \\
\hline
\end{tabular}
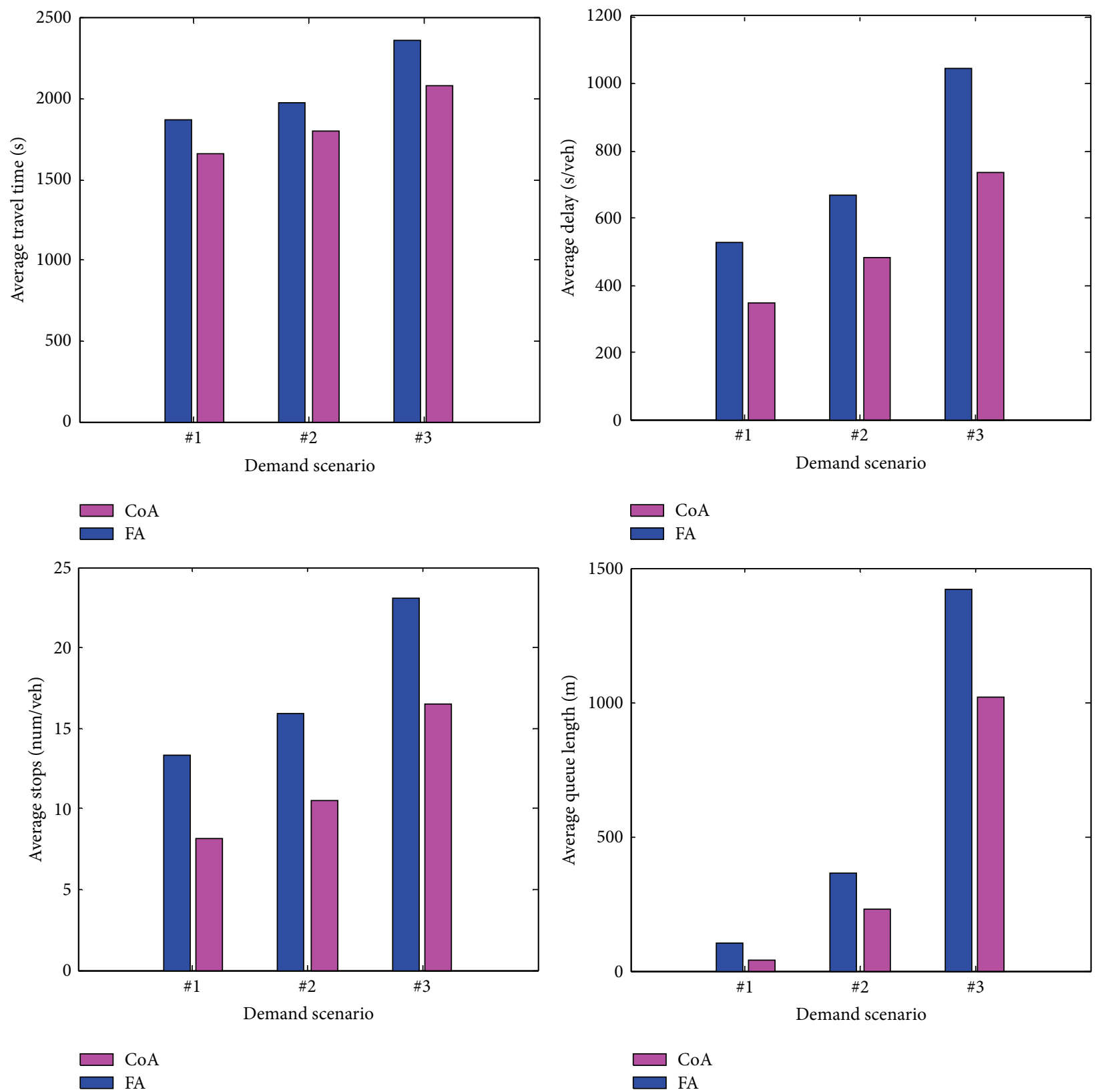

FIGURE 17: Comparison of network performance. 
CoA can greatly reduce the average delay, stops, and queue length.

In future, we will do further numerical and simulation studies with more complex and large-scale urban road networks aims to confirm the potential of the proposed method. For practical application purpose, research will continue on addressing the signal coordination of road networks under more realistic traffic conditions, such as transit signal priority; uncertainty traffic flow will be considered.

\section{Conflict of Interests}

The authors declare that there is no conflict of interests regarding the publication of this paper.

\section{Acknowledgments}

The work described in this study was supported by the National Natural Science Foundation of China under Grants no. 61374066 and no. 61473252 and the Fundamental Research Funds for the Central Universities of China.

\section{References}

[1] M. Papageorgiou, C. Diakaki, V. Dinopoulou, A. Kotsialos, and Y. Wang, "Review of road traffic control strategies," Proceedings of the IEEE, vol. 91, no. 12, pp. 2043-2065, 2003.

[2] I. Porche and S. Lafortune, "Adaptive look-ahead optimization of traffic signals," Journal of Intelligent Transportation System, vol. 4, no. 3-4, pp. 209-254, 1998.

[3] M. Ahmane, A. Abbas-Turki, F. Perronnet et al., "Modeling and controlling an isolated urban intersection based on cooperative vehicles," Transportation Research Part C: Emerging Technologies, vol. 28, pp. 44-62, 2013.

[4] S. Lämmer and D. Helbing, "Self-control of traffic lights and vehicle flows in urban road networks," Journal of Statistical Mechanics: Theory and Experiment, vol. 2008, Article ID P04019, 2008.

[5] X.-F. Xie, S. F. Smith, L. Lu, and G. J. Barlow, "Schedule-driven intersection control," Transportation Research Part C: Emerging Technologies, vol. 24, pp. 168-189, 2012.

[6] J. D. C. Little, "The synchronization of traffic signals by mixedintegerlinearprogramming," Operations Research, vol. 14, no. 4, pp. 568-594, 1966.

[7] N. H. Gartner, S. F. Assman, F. Lasaga, and D. L. Hou, "A multi-band approach to arterial traffic signal optimization," Transportation Research Part B: Methodological, vol. 25, no. 1, pp. 55-74, 1991.

[8] D. I. Robertson, "TRANSYT: a traffic network study tool," Report LR253, Road Research Laboratory, Crowthorne, UK, 1969.

[9] D. I. Robertson and R. D. Bretherton, "Optimizing networks of traffic signals in real time-the SCOOT method," IEEE Transactions on Vehicular Technology, vol. 40, no. 1, pp. 11-15, 1991.

[10] P. Lowrie, SCATS: A Traffic Responsive Method of Controlling Urban Traffic, Roads and Traffic Authority, Sydney, Australia, 1992.

[11] N. H. Gartner, "OPAC: a demand-responsive strategy for traffic signal control," Transportation Research Record, no. 906, pp. 7581, 1983.
[12] J. J. Henry, J. L. Farges, and J. Tufal, “The PRODYN real time traffic algorithm," in Proceedings of the IFAC Conference on Control in Transportation System, pp. 305-310, 1983.

[13] F. Boillot, S. Midenet, and J.-C. Pierrelée, "The real-time urban traffic control system CRONOS: algorithm and experiments," Transportation Research Part C: Emerging Technologies, vol. 14, no. 1, pp. 18-38, 2006.

[14] C. Diakaki, M. Papageorgiou, and K. Aboudolas, "A multivariable regulator approach to traffic-responsive network-wide signal control," Control Engineering Practice, vol. 10, no. 2, pp. 183-195, 2002.

[15] C. Stamatiadis and N. H. Gartner, "MULTIBAND-96: a program for variable-bandwidth progression optimization of multiarterial traffic networks," Transportation Research Record, vol. 1554, no. 1, pp. 9-17, 1996.

[16] Z. Tian and T. Urbanik, "System partition technique to improve signal coordination and traffic progression," Journal of Transportation Engineering, vol. 133, no. 2, pp. 119-128, 2007.

[17] Z. Tian, V. Mangal, and H. Liu, "Effectiveness of lead-lag phasing on progression bandwidth," Transportation Research Record: Journal of the Transportation Research Board, no. 2080, pp. 22-27, 2008.

[18] X. Kong, G. Shen, F. Xia, and C. Lin, "Urban arterial traffic twodirection green wave intelligent coordination control technique and its application," International Journal of Control, Automation and Systems, vol. 9, no. 1, pp. 60-68, 2011.

[19] L.-T. Lin, L.-W. C. Tung, and H.-C. Ku, "Synchronized signal control model for maximizing progression along an arterial," Journal of Transportation Engineering, vol. 136, no. 8, pp. 727$735,2010$.

[20] K. Lu, X. Zeng, L. Li, and J. Xu, "Two-way bandwidth maximization model with proration impact factor for unbalanced bandwidth demands," Journal of Transportation Engineering, vol. 138, no. 5, pp. 527-534, 2012.

[21] N. H. Gartner and C. Stamatiadis, "Arterial-based control of traffic flow in urban grid networks," Mathematical and Computer Modelling, vol. 35, no. 5-6, pp. 657-671, 2002.

[22] N. H. Gartner and C. Stamatiadis, "Progression optimization featuring arterial- and route-based priority signal networks," Journal of Intelligent Transportation Systems: Technology, Planning, and Operations, vol. 8, no. 2, pp. 77-86, 2004.

[23] B.-L. Ye, W. Wu, X. Zhou, W. Mao, and Y.-S. Huang, "A green wave band based method for arterial signal control," in Proceedings of the 11th IEEE International Conference on Networking, Sensing and Control (ICNSC '14), pp. 126-131, Miami, Fla, USA, April 2014.

[24] X. Zhou and Y. Lu, "Coordinate model predictive control with neighbourhood optimisation for a signal split in urban traffic networks," IET Intelligent Transport Systems, vol. 6, no. 4, pp. 372-379, 2012.

[25] B.-L. Ye, W. Wu, and W. Mao, "Distributed model predictive control method for optimal coordination of signal splits in urban traffic networks," Asian Journal of Control, 2014.

[26] R. Scattolini, "Architectures for distributed and hierarchical model predictive control-a review," Journal of Process Control, vol. 19, no. 5, pp. 723-731, 2009. 


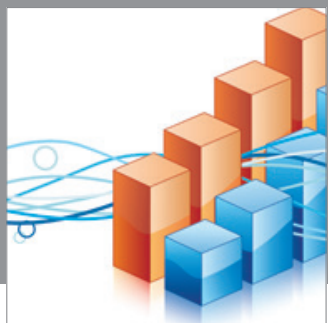

Advances in

Operations Research

mansans

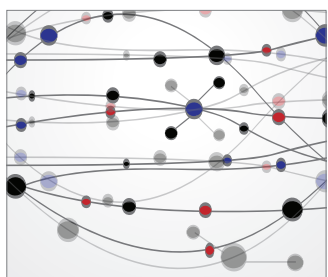

The Scientific World Journal
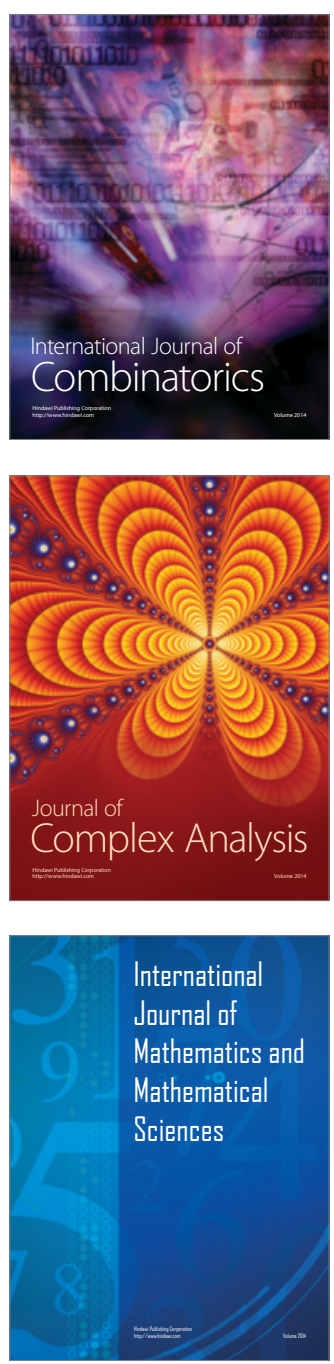
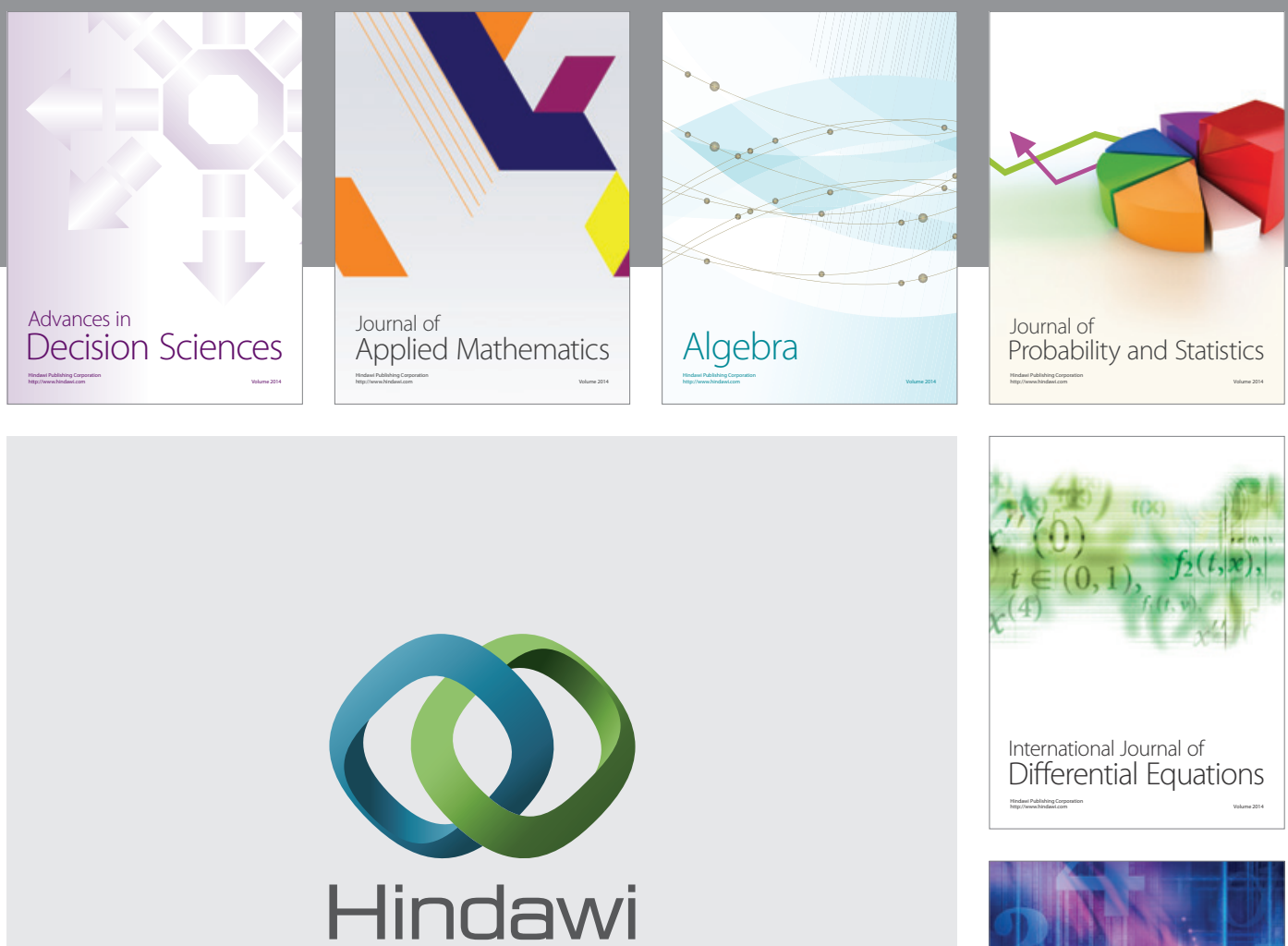

Submit your manuscripts at http://www.hindawi.com
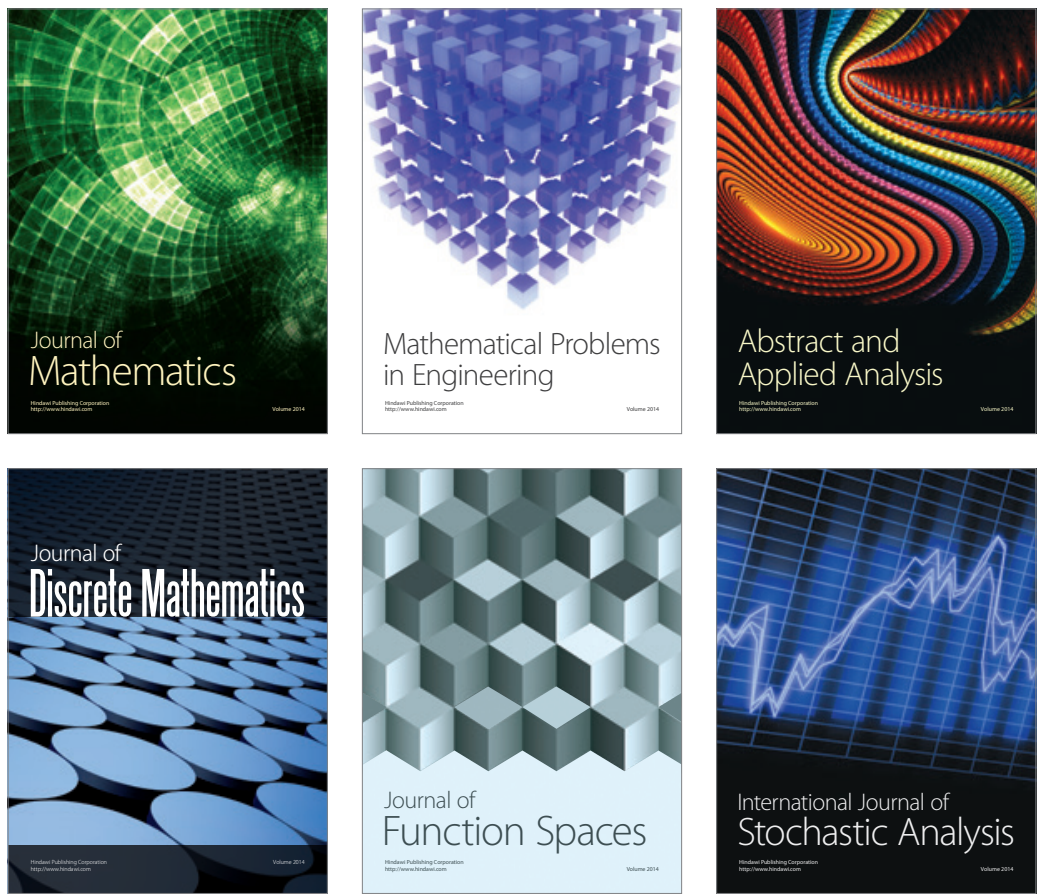

Journal of

Function Spaces

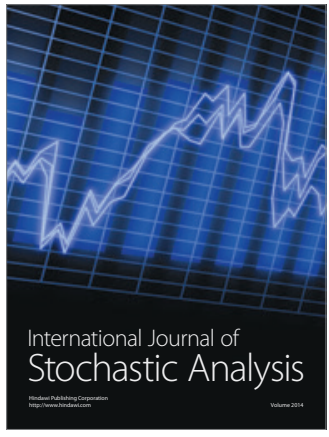

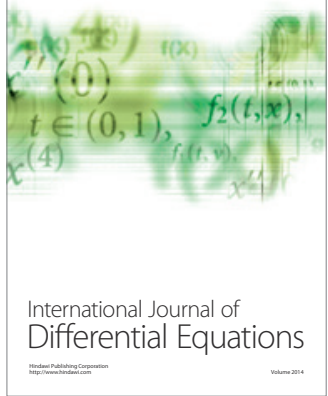
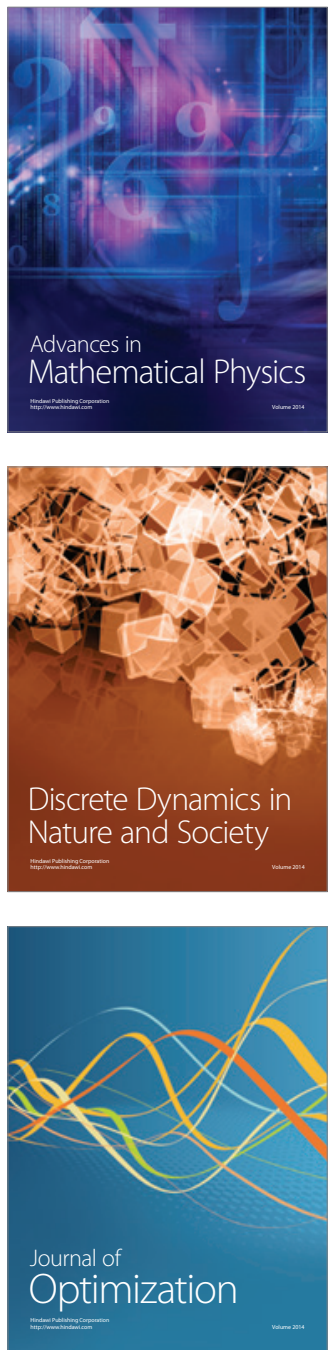